\title{
Discontinuous Precipitation of $\alpha$-Cr Phase in Alloy 33 (Cr-Fe-Ni-N)
}

\author{
J.C. Spadotto ${ }^{1}$, M.G. Burke², I.G. Solórzano ${ }^{1}$ \\ ${ }^{1}$ Department of Chemical and Materials Engineering, PUC-Rio, Rio de Janeiro, Brazil \\ ${ }^{2}$ Material Performance Centre, University of Manchester, Manchester, UK \\ *Corresponding author: juliocspadotto@gmail.com
}

Discontinuous precipitation (DP) reactions is a grain boundary (GB) diffusion-controlled phenomenon generating lamellar or rod-type equilibrium precipitates driven by a migrating GB that acts as a fast reaction path under the action of chemical and capillary forces, leaving behind a regularly spaced array of precipitates growing cooperatively from the supersaturated matrix. In multicomponent alloy systems containing both substitutional and interstitial solutes, DP usually is constituted of either Cr-rich $\mathrm{M}_{23} \mathrm{C}_{6}$ or $\mathrm{Cr}_{2} \mathrm{~N}$ lamellae [1,2]. Recently, $\sigma$-phase has been observed as the main precipitate comprising a DP colony in superaustenitic stainless steel [3]. The DP reactions are of practical interest because of its effect on corrosion and mechanical properties of alloys under service conditions [1,2].

The present investigation deals with the DP reaction in Alloy 33 with an austenitic matrix and nominal composition (wt. \%) 33Cr-32Fe-31Ni-1.6Mo-0.6Cu-0.4N. In order to understand the DP behavior upon isothermally aging at $800^{\circ} \mathrm{C}$, an experimental study has been conducted using scanning electron microscopy (SEM), scanning and transmission electron microscopy (STEM/TEM) with X-ray energy dispersive spectroscopy (XEDS), and electron backscatter diffraction (EBSD). Detailed microstructural analysis was performed using an FEI Talos F200A with Super X (4 Si Drift Detectors) and X-FEG for high sensitivity STEM-EDX spectrum imaging and analysis on electron-transparent thin-foil specimens prepared by the twin-jet electropolishing technique.

EBSD analysis have proved that the formation of a DP colony is strongly dependent on the misorientation between the FCC grains and can be rationalized by the differences in the energies of each segment of the boundary. A typical example of a DP colony frequently observed in Alloy 33 aged at $800^{\circ} \mathrm{C}$ for $10 \mathrm{~h}$ is shown in Figure 1, a bright-field (BF)-STEM of a DP colony containing some irregular precipitates at original GB position and lamellar precipitates growing into the FCC matrix. Also, another type of precipitate morphology and growth direction in relation to lamellar precipitates was detected within the DP colony. It was noted that this specific DP colony migrated into both sides of the boundary. STEMEDX maps obtained from this DP colony confirmed that: 1) the region swept by the boundary was Crdepleted, and 2) at least 3 different precipitates were identified within this DP colony. STEM-EDX data confirmed that all precipitates were $\mathrm{Cr}$-rich. However, at original GB position some precipitates contained increased levels of Si and Mo (white arrows), in addition to another Mo-rich precipitate (yellow arrow). Furthermore, Si-enrichment was detected at the DP reaction-front (red arrow), also confirmed by XED microanalysis.

The centred dark-field (CDF)-TEM image in Figure 2 shows the lamellar discontinuous precipitates indicated by the box in Figure 1. From the SAED pattern shown in the insert, these precipitates were identified as the BCC $\alpha$-Cr phase. The corresponding XED spectrum shown in Figure 2 indicates this precipitate is $\mathrm{Cr}$-rich but also contains $\mathrm{Fe}$, in addition to $\mathrm{Mo}, \mathrm{Ni}$ and $\mathrm{Si}$. In multicomponent alloys, this phase has been reported to form after long-term aging in several morphologies (intra and intergranular modes) and in the vicinity of $\delta$-phase [4]. However, lamellae of the $\alpha$-Cr phase that has constituted these DP colonies have only been reported to form in binary $\mathrm{Ni}-\mathrm{Cr}$ alloys [5][6]. 


\section{References:}

[1] Zheng, L., et al. Materials \& Design, 78 (2015), pp. 42-50.

[2] Simmons, J.W. Metallurgical and Materials Transactions A, 26 (1995), pp. 2579-2595.

[3] Zhang, S., et al. Materials Characterization, 137 (2018), pp. 244-255.

[4] Gailian, W. et. al. Materials Science and Engineering A, 358 (2003), pp. 71-75.

[5] Keskar, N., et al. Metallurgical and Materials Transactions A, 48 (2017), pp. 3096-3107.

[6] The authors are grateful to support of Brazilian Funding Agency-CNPq, Brazil.

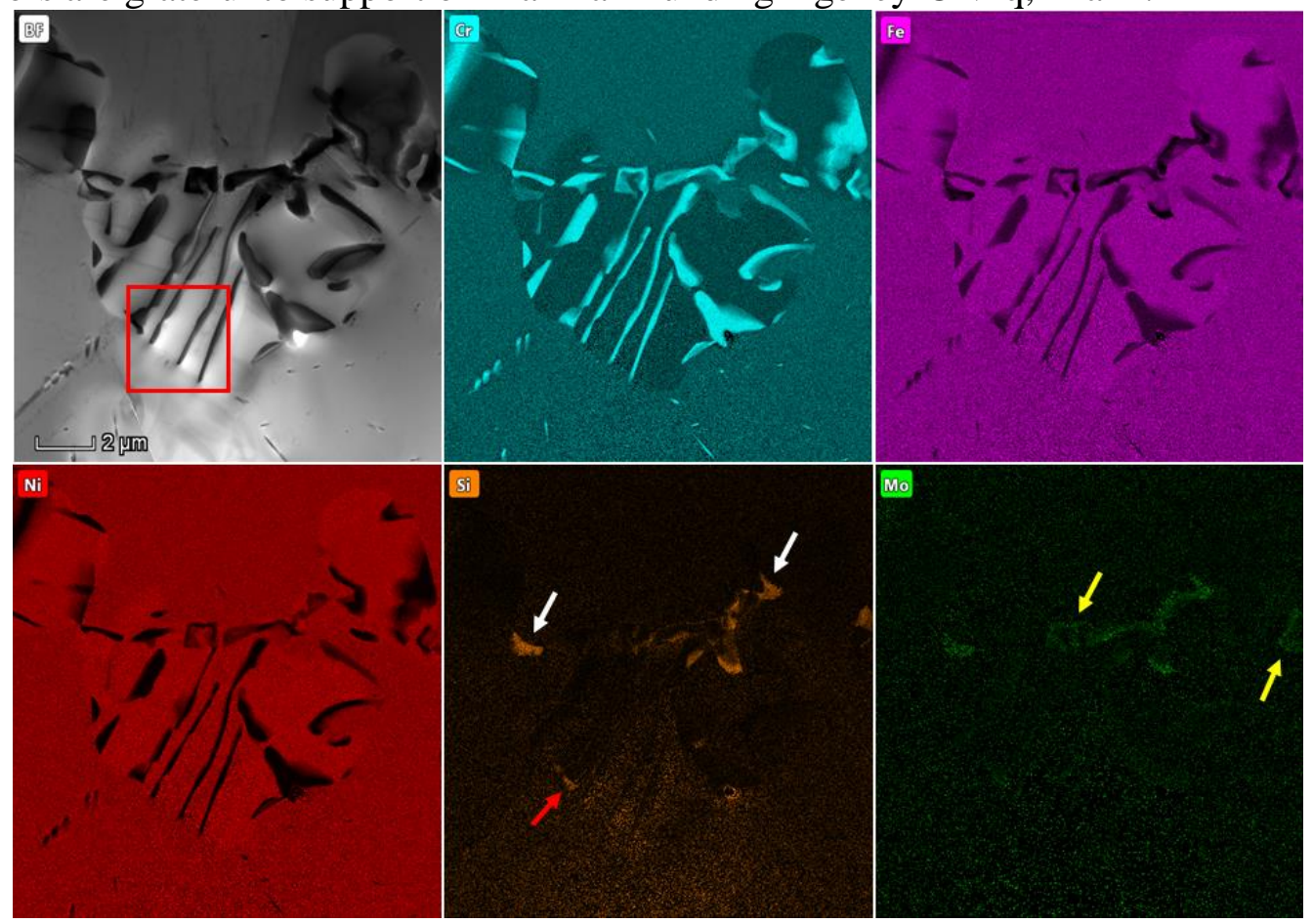

Figure 1: BF-STEM image of DP colony and corresponding STEM-EDX maps showing the partitioning of $\mathrm{Cr}, \mathrm{Fe}, \mathrm{Ni}, \mathrm{Si}$, and Mo.

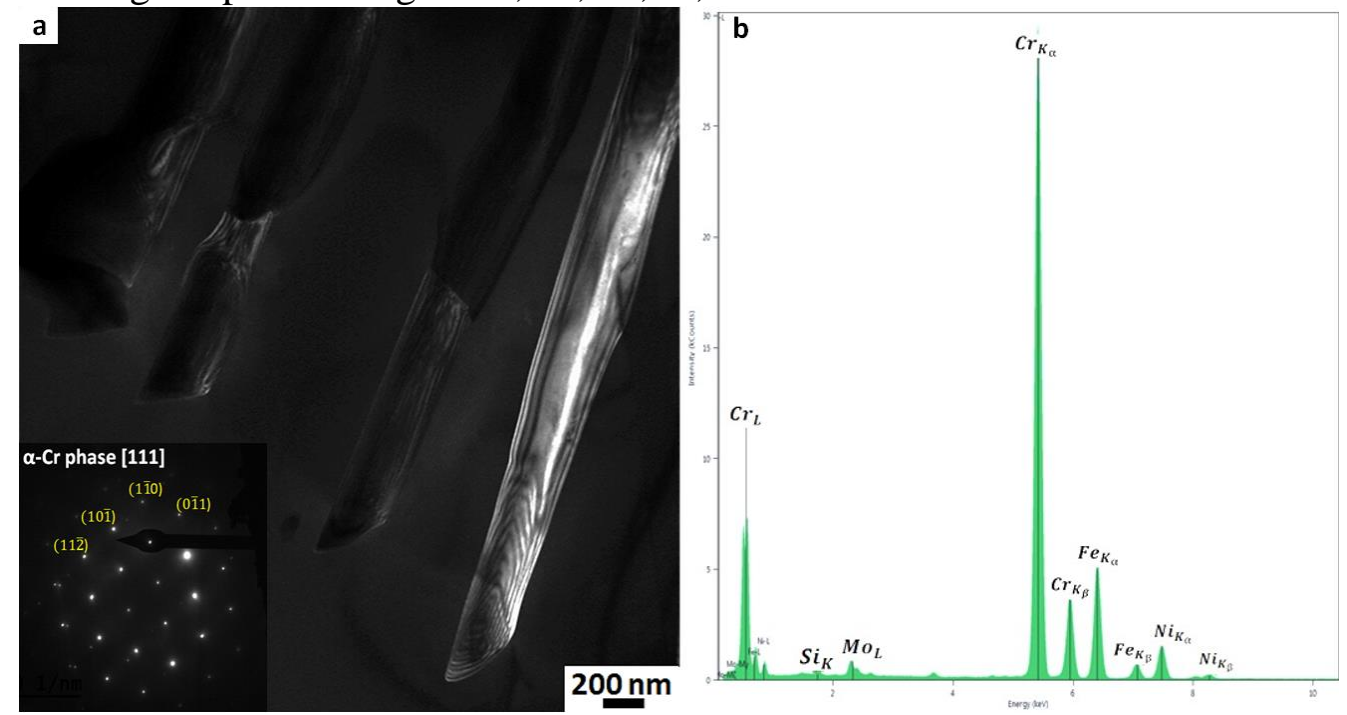

Figure 2: (a) CDF-TEM image of lamellar precipitates indicated by the red box in Fig. 1, that were identified as $\alpha-\mathrm{Cr}$ phase with $\mathrm{BCC}$ structure; (b) XED spectrum from lamellar precipitates shown in (a). 